\title{
1. The new global politics of research
}

What is the most central issue defining our time? A few contenders are climate change, social inequalities, technological change and digitalization, sustainable growth, migration, poverty, unemployment, populism and national security. The issues listed all share some basic characteristics: they are complex, political, and social, and open to a wide variety of interpretations. Underlying all of them is also, in one way or another, knowledge. Knowledge can be deployed not only to articulate those issues and their underlying causes, but also to outline their management and resolution. Knowledge is not merely a servile and versatile instrument to tackle complex problems; it is also the subject of contestation and social strife, and is mobilized for quite different aims. Knowledge may be ignored or contested, used for oppression or liberation. Knowledge is a public good, widely available and circulated to benefit humankind, but also the object of intense private appropriation to create wealth and accumulate power - or worse. Knowledge is institutionalized in different ways: as parts of research and education, in transmissions and in popularization; as popular slogans but also as intricate and esoteric pieces of information.

While these forms are ideally related to one another, knowledge appears in forms that are often incompatible - the tentative results of a scientific paper often appear as truths in popularizations. Knowledge is cherished as a voice of reason in an era of "fake news" and blatant expressions of intertwined power and ignorance. But knowledge itself is not certain or undisputable; in a global knowledge system in constant expansion - in terms of people, activities and organizations struggling for attention and visibility - claims of certainty and validity are often overblown.

There are many ways to disentangle the complexities of knowledge in society: studying processes of scientific validation, the formation of scientific expertise, the systemic relations between knowledge, markets and states, or the use of knowledge in social conflict are some dominant themes in the contemporary literature. A significant issue is the institutionalization of knowledge: how it is organized, funded, managed and used. This institutionalization in turn takes different forms and influences 
many of the critical issues raised above, such as: how knowledge shapes markets and firms (Nelson and Winter 1982); how it intersects with political processes and the formation of states and other political entities (Rueschemeyer and Skocpol 1996); and how it shapes, and is shaped by, social categories such as class and gender (Orloff and Palier 2009).

A key aspect here is, of course, the regulation of research itself, which plays a pivotal role in the generation and utilization of knowledge in society - in markets, in politics and in social relations. Ever since research became an organized activity, with adjacent forms of internal communication and societal articulation, it has been subject to governance and regulation in different forms (Shapin and Schaffer 1986). Regulation is intended to highlight how different conditions, demands and expectations on and for knowledge are aligned (or not) in different contexts and different periods in time. Early on, knowledge was largely deployed to expand and secure the internal and external boundaries of the state. With the advent of organized capitalism in the 19th century, social order and economic extraction became the leading themes of knowledge regulation, themes that remain central to the practice and governance of knowledge - with new dimensions and issues added later as a response to social, economic and political pressures and processes (Elzinga and Jamison 1995).

The configuration of regulation models can therefore be expected to be relatively stable over time, but also with considerable variations between countries in the exact form of that configuration. Such regulation models therefore configure the relationship between research, politics, markets and society on a general level, but with considerable space for variations between contexts. This book aims to make sense of how research and knowledge are governed in contemporary society, and the ambitions but also contradictions and incompatibilities that ensue in different contexts.

\section{SCIENCE: STILL AN ENDLESS FRONTIER?}

What, then, are the contemporary conditions for research? A first observation is that it has become a dominant part of modern society. Currently, more than 20,000 research institutes and universities exist worldwide. Global expenditure on science is difficult to measure, but a recent estimate is that annual investments in research amount to about 1.7 trillion US dollars (National Science Board 2016). The number of researchers in the world is over 10 million, and in some highly developed countries almost 1 per cent of the adult population is employed as scientists. China and the US dominate the labour market for scientists, 
each with around 1.5 million scientists in employment. China has seen a particular spike, with a five-fold increase in its research cadre between 1996 and 2016. If wider definitions are deployed - including all those working on tasks that demand an higher degree in science - the figure for the United States alone rises to over six million, with a similar figure for China (ibid.).

Another critical factor for the regulation of knowledge is the form and shape of the regulation node - that is, the state. The state may be seen as an ensemble of organizations and activities that align and combine contradictory social and economic forces in society (Jessop 2016). The state is therefore a critical intermediary between different forces and interests, such as those of companies, political parties and civil society. In its post-war incarnations, research policy held a supportive position in relation to other areas of public engagement - defence and energy policy in particular. Research served limited but critical functions for the cycle of economic growth, political mobilization and social reproduction in this period, a cycle that was instead primarily fueled and underpinned by other forces such as national security and the regulation of largely domestic markets (Boyer 1990).

With the deregulation of financial markets in the 1980s, the rise of a neo-liberal economic and political ideology stressing the virtues of free markets, the fall of the Berlin Wall and the integration of China and East Asia into the world economy, the conditions for states and governments changed fundamentally into what has been labelled a "competition state". In this current state model, growth and social integration are driven less by the (relatively) harmonious interaction between supply and demand of standardized goods for relatively homogeneous domestic markets, and more by the imperative of competitiveness on global markets - which in turn is contingent upon, among other things, the production, dissemination and utilization of knowledge. The current mode of growth therefore presents the state with a different set of expectations and demands than earlier economic growth models had in their time: rather than emphasizing fixed and stable demand, the state is expected to enable the productive and flexible use of resources for fluctuating markets.

The "scientific state" that was heralded already in the 1960s (Price 1965) may therefore finally have come to fruition, in the form of a state that socializes costs and risks of corporate innovations to enhance the competitiveness of firms on global markets, and that ensures that higher education and research are aligned with the existing and future interests of civil society and the economy (Hilpert 2002; Mazzucato 2011). This tendency includes the governance of the state itself, which has become the subject of scientific techniques to understand and master its modes of 
operation - "second modernization" (see Böhme 1992; Latour 2003). While the intersection between science and the state is certainly not a novel phenomenon but rather a historical constant, the "competition state" as outlined here is fraught with many constraints that in themselves call for policy advice and analysis. This also includes of course science policy studies, which have been increasingly concerned not only with how economic growth models shape policy forms and directions (Nelson 1993; Larédo and Mustar 2001), but also with how novel forms of risks and uncertainties can feed back into models of policy formation and implementation (Rip 2016).

Another aspect is geopolitical. Up until around 1980 the global knowledge system was dominated by the USA in terms of expenditure, visibility, networks and patterns of exploitation. Interactions between North America and Western European and the Soviet Union and China were minimal and carefully managed, and the knowledge system was polarized along geopolitical lines. The transformation of the state is paralleled with a radical redeployment of global relations and forms of interaction, visible also in science and innovation (Castells 1996, Wagner and Leydesdorff 2005). The current knowledge system is, for want of a better description, less centralized and more globalized in the sense that the distribution of resources is more variegated and the opportunities for pursuing research and innovation beyond the metropoles have grown (Hollingsworth et al. 2008; Lee 2013).

These tendencies notwithstanding, it is not a "flat" system: the historical dominance of the United States still shows in the constitution of the scientific system, which gives countries with historical, institutional and linguistic ties to the US advantages over those with only limited connections (Gingras and Khelfaoui 2017). Hence, globalization in itself is fraught with contradictions, and the placid expectations of a global governance system in which these tensions and patterns of domination will be reconciled appear overly optimistic, also in the area of knowledge (cf. Kuhlmann 2001). Indeed, it may very well be the case that the ideals of globalization and "global governance" will give way to geopolitics and widening relations between nation-states once again, not in the form of explicit military conflicts as in the post-war period, but possibly over issues such as intellectual property, communications and mobility (cf. Hirst and Thompson 1995). 


\section{WRITING THE HISTORY OF RESEARCH POLICY}

This is certainly not the first, and for that matter will not be the last, attempt to sketch tendencies in the governance of knowledge. Already 50 years ago, science journalist Daniel Greenberg (1967) juxtaposed "science" and "politics" in a classic study. In Greenberg's reading, even the purportedly "purest" of scientific endeavours was entrenched in politics, and the boundaries between the two could not be drawn easily: scientists were engaged in essentially political struggles to expand the public funding of their work, while politicians with similar drive sought to enlist science and scientists in their policy ambitions, be they in defence, energy or health.

What seemed frivolous 50 years ago has become commonplace, to the extent that, to paraphrase Carl von Clausewitz's dictum on war, science is politics by other means (see Latour 1993). Science not only intersects occasionally with politics; the relations are seamless, and it is well-nigh impossible to tell where the one begins and the other ends. Indeed, this observation is in itself highly political, and contested in both form and content.

The changing relation between science and politics is manifested not only in the enormous proliferation of studies of the connections between science and politics. It is also shown in politics, where the number of measures and instruments aligning the relation between politics and science has multiplied and continues to grow.

\section{Growth and Institutional Change}

At the time Greenberg was writing, science was a rapidly growing undertaking, but the paucity of growth had not yet been matched by institutional realignment on a broader scale. At this time, the governance system in terms of agencies, programmes and plans was rather rudimentary. Indeed, among the big issues of the day was how science could and should be governed; how funding was to be allocated; how accountability could be ensured; and, more generally, how the fruits of science could be reaped. Even the issue of language was open to debate. Even though Greenberg used the term "pure science" - which was connected to a popular understanding of science as an independent activity, beyond the reach of social, political and economic interests (cf. Shapin 2010) - it would be more reasonable to claim that he studied "basic research" in its political setting (Kaldeway and Schauz 2017).

"Basic research" as a denominator was insufficiently defined in terms of outputs and directions to be palatable to the academic system, but 
sufficiently focused to merit and garner political backing and recognition ("research" indicating an organized activity of some scale and professionalization). This combination of opacity and expectations opened up the way for mavericks like Greenberg's fictional character Dr Grant Swinger to straddle boundaries and explore funding opportunities for purportedly endless societal benefits and for the furthering of human knowledge.

The institutional lacuna has since evolved into an extensive branch of government, both in terms of funding and in terms of administrative interventions - such as written reports, policy committees and the like. Science policy is no longer a loose assembly of activities linking scientists to the purses of government in an on/off manner. Instead it is an institutionalized system for the governance of research for a wide variety of purposes, with links not only to economic policy, labour market and employment, innovation and a wide variety of other issues, but also to an increasingly dense and complex regulation of the practices of research. It is also an issue of considerable social concern, with numerous channels for debate and controversy around the interaction between social interests and scientific purposes, be they related to ideological concerns or social movements.

Science is also framed as an economic instrument, assessed and valued in both qualitative and quantitative terms for its consequences for economic growth and entrepreneurship. The simultaneously hierarchical and opaque politics that characterized the 1960s - when policy anarchy in small networks co-existed with major interventions and investments, seemingly without coordination or linkages - have evolved into a rather well-defined policy domain, albeit a domain with many entry points and a wide range of interested parties.

\section{A New Social Contract}

Greenberg's study was published on the cusp of several profound transformations of the relationship between science, politics and society. The social upheaval of the late 1960s ushered in a critique of the social consequences of academic research, its connections to defence and economic dominance, and the lack of articulation with pressing social concerns as expressed by science-critical movements (Blume 1974; Böhme 1992). Science policy quickly evolved from a secluded and elitist area (dominated by military, industrial and academic echelons) into a contested area, and the governance of knowledge production and use was subjected to a far more transparent, and therefore also conflictual, model. The dominant discursive representations of research of the post-war period - Big Science, basic research, pure and disinterested science - 
were largely superseded by a vocabulary that drew more on the accountability and utility of research: applied, strategic research, sectoral knowledge, democratic innovations, open universities, and similar. Science was interwoven in a new web of dependencies and relations, and its capacity to resolve societal contradictions came to the fore.

Up until about that time, the early 1970s, leading science students had primarily investigated the self-regulatory mechanisms of science and its interplay with other societal spheres in the emergence of modern society (along the lines outlined by Merton 1973). While different in their approaches and interests, all were concerned with the functions and governance mechanisms of a growing scientific system, highlighting (and postulating) the autonomy of the academic system, with industry and society absorbing discreet scientific facts. Only very rarely were the connections between science and society taken up, and the dominant theme was instead how the scientific system was reproduced over time by the scientific community itself. In line with the then fashionable structural-functionalist theory, society was understood as being made up of autonomous but interacting sub-systems; hence, the research system (cognitive in structural-functionalist terminology) was seen as separate from the three other spheres in society: culture, polity and the economy. Daniel Bell (1973) was one of the first macro-sociological students to take an interest in the increasingly professionalized and multifaceted "research and development system" (R\&D system), a system of growing complexity with a multitude of dependencies and relations between science, politics and the economy. Bell's rather agnostic observation that science actually equalled power and dominance became the starting point for the first generation of modern science policy studies, which took up the hitherto largely neglected issues of values, interests and power in and around science.

The analytical shift - at around 1970 - emerged in parallel with the rise of a new generation of science policy instruments, and the redirection of older ones. The technocratic policy paradigm of the 1950s and 1960s was widened, not only to include areas of the developing welfare state but also to extend the social basis of policy-making, making way for the inclusion of trade unions, social movements and others marginalized in policy-making in the post-war period. This was an era of rediscovering the political and articulating a critical perspective on science and technology in society rather than an autonomous and self-regulating sphere as depicted by Merton and his school of science students. The neo-Weberian and neo-Marxist turn that took place stressed dominance and authority in and around science, articulating the voices and interests of marginalized groups (Rose and Rose 1971; Blume 1974). Issues of 
gender, class and international exploitation and dominance took hold of the field, at the expense of functionalist and systemic accounts.

\section{The New Politics of Research}

In the late 1970s, as part of a disenchantment with the "political turn" in science studies, a phase of integrating philosophical, anthropological and linguistic perspectives in the study of science ensued, a period which is still vital. It included studies of science as a practice, as well as the fluctuating and porous boundaries between researchers, technologies, material, users, politicians and other actors. The philosophical and anthropological turn highlighted how the political was not primarily about "knowledge conflicts" and social power relations in dedicated political forums. Rather, it was enmeshed in (almost) everything in the daily lives of women and men; politics emerged as a ubiquitous rather than a compartmentalized aspect of knowledge in society.

The entire range of studies along these lines is impossible to reconstruct here; but suffice to say that they, in all their variation, seldom or never deal directly with the constitution and implementation of science policy programmes; politics resides elsewhere in their reading of the world. Politics in the literal sense has later on returned to science, via the so-called science wars and the confrontation between scientists and science students on how science should be understood (Collins 2014). This has also taken the form of studies of the politics of participation, of scientific citizenship, in critical debates on what science is and what it represents in society (Frickel and Moore 2005).

The last decades have also seen a surge in studies of the role of science in creating normality, gender and individual identity, and of how technologies have been deployed to increase self-control and the governance of mind and body (Rose 2000). This evolution did not take place in a political vacuum, of course: some aspects of this are the increasingly tense debates on the accountability of science in society and the social consequences of new technologies; another aspect includes the interface between technology and humanness (stem cells, genetic engineering and so on). Other factors that shape contemporary debates on science include the articulation of science with the economy and the increasing financial pressure on (public) universities and the marketization of academic knowledge production (Mirowski 2011).

Beneath the surface of the "new politics of research", the old politics (concerned with resource and power distribution) has remained an issue which has engaged some - primarily eminent and seasoned - scientists. John Ziman (2000), physicist turned social scientist, has highlighted the 
integration of the scientific enterprise into the social fabric, where previously autonomous norms and procedures were opened up - from funding to ethics - and subjected to political decision-making rather than professional consideration alone. In response to an ever-increasing complexity of research-funding instrumentation, a literature on the steering capacity and steering outcomes of research policy has ensued (Braun 1993; Whitley and Gläser 2007). It deals with the organizational dimension of knowledge, particularly framed in contractual terms and on the negotiations enmeshed in various models of supporting science. It has a focus primarily on funding as a contractual arrangement between patrons and agents, and of how these arrangements orchestrate and negotiate risks and gains between the polity and the scientific system when research policy programmes are devised, implemented and assessed.

More recently, studies of the organizational dimension of science have expanded into the management and work organization of teams and other collaborative mechanisms, not only as a critical level in the performance of research but also as an increasingly salient node in combining power, resources and networks (Heinze et al. 2009). The rich literature on higher education and university governance has also recently began aligning with the dynamics of science and technology, as the interplay between management and governance of universities and the dynamics of knowledge production is increasingly critical for both (Whitley and Gläser 2007; Labaree 2017).

All in all, the politics of research is a variegated field and, while the "old politics of research" no longer forms the centre of the area of science and technology studies, the issues of the regulation, funding and power in and around knowledge remain key and critical issues - more so today than just a few years ago.

\section{WHY THIS BOOK?}

In the first handbook of STS from 1977 - when the abbreviation stood for Science, Technology and Society and not, as today, for Science and Technology Studies - the field was entrenched in politics and political perspectives (Spiegel-Rösing and Price 1977). Among the issues taken up in the handbook were: social and political critique of science; science and its articulation with the international political system; scientific and technical development and its impact on economic growth; and science and the military. Significantly, there were only a few contributions on scientific and technological practices, and those dealt primarily with the normative and collaborative elements in science. Hence, critique and 
debate of political processes were still the overarching themes of the field, a legacy of the field's emergence concomitantly with the "science critique" of the late 1960s, even though many of the students had become seasoned academicians instead of passionate activists by the time the handbook was published.

While the handbook was one of several signs of the institutionalization of the field (others included the rise of $\mathrm{PhD}$ programmes in the field and the inception of a society for social studies of science), it still received critique at the time - especially from scholars in neighbouring fields like sociology, who viewed its disparate orientation as an indication of the fragility of the field, overly focused on "issues" and "problems" rather than on concepts and theories. This critique, in conjunction with the process of professionalization associated also with interdisciplinary and problem-based research fields, paved the way for the next step in the development of STS, where the focus shifted instead to the practices and forms of social order that science contributed to producing (Pestre 2004).

Whatever the merits and content of the critique, and despite the relative stability of the nascent field, the political background of STS still loomed over its students and their selection of study objects. Political problems and issues were indeed central also to STS at the time: the allocation of power and resources, and the interplay of science and technology in various political domains figured prominently. Hence, one of the major influences shaping the STS field was the political - whether it was Marxist in orientation, global inequalities and economic development, feminist or Weberian; power and influence in the shaping of science and technology; or in the shaping of society via science and technology. There were signs of a divergence and a growing marginalization of the political already in the handbook. One of the editors of the STS handbook, distinguishing between Science and Technology in Society (STS) and Science and Technology Policy (STP), pointed to the relative separation of the areas, a point later substantiated and underscored by the (virtually non-existent) relationship between STS and policy studies since (Spiegel-Rösing 1977; see also van den Besselaar 2001; Martin et al. 2012).

\section{THE END OF POLITICS?}

As STS matured, it did so with a radical redefinition of the "political" and the connection between the field and policy processes more broadly. The political aspects dealt with within STS studies were increasingly: the micro-aspects (negotiating claims of success and stability in research in 
the laboratory); the workings of tacit knowledge networks for the development and understanding of codified knowledge; and the interplay between technical knowledge and the political interplay between public and scientific/technical communities, and in particular how scientific results are made credible in the eyes of the public and how scientists and science gains credibility (Martin et al. 2012). It might even be said that the entire business of STS - at least in its actor-network iteration - aims at subverting notions such as "society", "politics" and "economics", or, for that matter, "nature" (Latour 2004).

STS studies were increasingly concerned with cognitive issues and the intertwining of knowledge production and social contexts, leaving the traditional political issues to be managed by STP scholars. STP scholars, on their side, operated with a rather straightforward orientation towards supporting policy-makers and policy constituencies with studies of how research (and technology) were being supported and organized, and with what consequences. With the advent of competition-based policies and the surge in innovation perspectives of how economic growth and social integration might be attained, the engagement of STP has mostly merged with innovation studies, fostering a new policy (and analytical) field innovation policy studies (Martin 2016a).

While the concomitant orientation towards the social and the instrumental has shaped the entire field of science studies, according to Martin et al.'s overview of the STS field (2012), only one "pure" policy study (Dickson 1984) made it onto the list of influential studies in the area. This cleavage is still a central feature of the social studies of science, leaving several important areas of the interface between research and politics largely unexplored.

\section{KNOWLEDGE POLICY: WHAT IT MEANS}

I aim to fill this void by pointing at the various facets of the political that intersect with research, facets that are not necessarily easily reconciled or even compatible but which nevertheless operate in parallel. I will organize them as constituent parts of a "knowledge policy", which articulates with different facets of knowledge and politics in contemporary society: state formation and nation-building; metagovernance and the alignment of different parts of the state, the market and economic goals; and, finally, the connection with social forces and social mobility.

The first aim of this book is therefore to describe and outline the relations between science and politics today: how the relations are 
configured; their political foundations; relations with scientific organization; and their general articulation with the directions of the societies in which they evolve. Hence, I set out to clarify what the politics of science actually represents today, in a manner similar to Greenberg's pioneering study 50 years ago.

The second aim is historical. With hindsight we now see that Greenberg's (1967) stocktaking came at a critical juncture in relations between science and politics. His book signalled a new and more agnostic view of science and its role in society, where massive investments might not only level out but actually contract and fluctuate, depending on social, political and economic interests. The post-war dynamics of continually growing investments were beginning to wear off, and the expectations of returns, impact and transparency were growing. The unstable relationship between the "warfare-welfare" state - with ever-increasing aims to secure international security and domestic social and economic stability and the increasingly complex system of scientific activity were in need of more robust and resilient institutional underpinnings. The complexity that Bell (1973) described some 40 years ago has grown and continues to mutate, with the parallel rise in economic relations, social expectations and interpenetrations with other policy spheres.

A third aim of this book is political. STS has moved from the margins of academia - with an activist twist - into a sizable industry of conferences, journals and $\mathrm{PhD}$ and Master's programmes. But that process has, arguably, reduced the intellectual traffic between science and technology studies and other fields such as sociology, political science or economics. Arguably, it has also lessened the interactions between academic research on science and technology and movements and policy circles. Instead, the area has developed its specific intellectual programmes, ambitions and networks, and may appear somewhat selfcontained - at least in its articulation with politics and political forces.

This has partially drained the field of influences from other areas; and, more importantly, it has cut off some of the energy that changes in science and technology bring to other social scientific areas. The classic texts in the field were all deeply rooted in broad public debates, Greenberg (1967) being a pre-eminent example. Sociological studies like those of Robert Merton also aligned social science and politics via midrange concepts such as reward systems and norms, and how these shaped individual and collective trajectories. Only a few social scientists today engage seriously with science and technology in their political and organizational settings, and the disjuncture of students and practitioners of science has become a growing concern (Collins 2014). 
It is striking that historical narratives informed by critical theoretical concerns are largely absent in the science policy literature, which is instead focused on various technicalities in the governance and regulation of research. The main supply of policy narratives today, which this book draws heavily on, comes from science journalists such as van Noorden (2012, 2015, 2016). While these provide rich and well-researched insights into policy processes, they are - quite naturally - shaped by a communication forum that puts a premium on access and speed.

As a small but not insignificant caveat, I do not reduce the dynamics of science to political processes alone. As an illustration, the rise of molecular biology drew not only on a combination of intellectual mobility from physics into the wider domain of biology, but also resource mobilization within and beyond the academic community - the state and private interests (Mullins 1972; Morange 1998). This rise of molecular biology - which has transformed biomedicine and contributed to the phenomenal rise of the biotechnology industry - cannot be understood without the context of a growing societal engagement in science; nor can it be reduced to merely an epiphenomenon of the growing rationalization and complexity of society and politics in the post-war period. They shape one another in an interdependent process.

\section{THE POLITICS OF RESEARCH: SOME STARTING-POINTS}

This is a macrosociological study which aims at an integrated understanding of science in its contemporary societal context. That context, which is still very much evolving, is a complex melange of marketization, globalization and symbolic and knowledge-intensive forms of accumulation, and of growing social and political expectations of knowledge as a productive force.

Macrosociology can be done from different starting-points. Its classical focus was on a specific process and its historical evolution and social forms: rationalization, division of labour and social conflict were among the identified foundational aspects of studies of conflict and cohesion in society (Turner 1999). More recent studies emphasize aspects such as the critical importance of gender relations and power (Walby 2009); forms of culture and social orders (Alexander 2013); the transmission and utilization of information (Bell 1973; Stehr 1994); or the intersection between politics and economics (Beckert 2002). The starting-point here is that knowledge and its societal articulation are fruitful for a macrosociological study. Knowledge operates in several dimensions in parallel: for 
example in micro-environments (libraries, laboratories and so on) or in organizational settings (universities, laboratories, institutes, companies, government agencies, organizations and so on), with their specific cultures and reward and promotion systems. It also aligns with funding mechanisms, with nation-states and their knowledge interests, with global relations and with markets. Knowledge also operates in different but intersecting contexts: it is an activity with its own specificities, forms of communication and validation which at the same time evolves in a context of organization, funding and other forces.

\section{Knowledge and Politics}

This book is therefore an elaboration of a political understanding of knowledge which takes into account the multifaceted articulations of science with the political. The "political" is understood not as confined to policy in the more restricted sense; rather, it is an extended understanding taken to be the negotiation with science over money, power, influence and other facets of the distribution of power in society.

The starting-point of this book draws on both the aim to elucidate some conflictual and unstable tendencies in contemporary society: that knowledge is subject to political, social and economic interests; and that its regulation therefore straddles different guiding principles. I aim to describe and decipher these contradictions, and how they have evolved and played out in different contexts.

Political economy is another intellectual anchor of this study, in the sense that it aims to align the anatomy of the political - parties, social movements, the state apparatus and its different organizational facets (including research policy) - with the structure of the economy in an interdependent manner where markets and politics are seen as co-evolving (Boyer 1990). A particularly important trait of political economy is the identification of societal ruptures and crises, and explaining how these evolve and how different solutions and remedies emerge to mitigate the contradictions between the social and the economic (Jessop 2016). This emphasizes the importance of relating the specificities of policies in the areas of science, technology and innovation to patterns of economic development. By "the political" I mean politics at the following levels:

- the internal politics of science - the power structure of scientific work (in its organizational and cognitive dimensions);

- the institutional structure of science (its settings and their power dynamics); 
- the regulation of science (funding systems, the regulation of conducts and practices, support of working modes including networks and collaborations); and

- the societal embeddedness of science (its articulation with markets, politics and social forces more generally).

The intention is to capture all these dimensions in an understanding of the dynamics of knowledge. I do not seek to unravel any causal linkages or mechanisms, only to pinpoint various contingent relations between them and broad patterns of power relations in and around knowledge. My focus is on systematic relations between modes of regulation in and for science. I locate the power of science in nested relations between power structures on different levels, including the power structures within scientific fields themselves. The starting-point is, however, institutional: I search for interrelations between scientific practices, their institutional settings, organizational networks and location within a broader framework of resources, identities and relations.

\section{OUTLINE OF THE BOOK}

My starting-point is historical, institutional and comparative: Historical in locating the tendencies in time and how they have evolved. Institutions and institutional settings are at the forefront of this study, as defined by the interplay between the political system, social interests and economic processes. I aim to elucidate institutional configurations and how they evolve over time, and how they reflect social mobilization and interrelations with other spheres of society. Comparative, finally, in that I study the composition and evolution of the politics of research in different countries to see how general tendencies play out in different contexts (Box 1.1). Here I have chosen to study the dominant powers in the knowledge system, namely:

- the US, obviously, which formed the basis of knowledge development throughout most of the 20th century, and which still is leading - if no longer in absolute terms;

- Europe, where science originated as an organized activity and which, in its north-western parts at least, is still one of the main sites of knowledge development and utilization; and

- Asia, where China and India stand out not only for their sheer size but also for their growing commitment to science as productive force, particularly China. 


\section{BOX 1.1 COUNTRIES UNDER STUDY AND THEIR R\&D INTENSITY}

China - R\&D expenditure $2.1 \%$ of GDP (of which $80 \%$ is corporate)

India - R\&D expenditure $0.7 \%$ of GDP (of which $50 \%$ is corporate)

United States of America - R\&D expenditure 2.8\% of GDP (of which $80 \%$ is corporate)

United Kingdom - R\&D expenditure 1.7\% of GDP (of which $65 \%$ is corporate)

European Union - R\&D expenditure: $2.0 \%$ of GDP (of which $65 \%$ is corporate)

Hong Kong - R\&D expenditure $0.7 \%$ of GDP (of which $80 \%$ is private)

Singapore - R\&D expenditure $2.0 \%$ of GDP (of which $65 \%$ is corporate)

\section{FOUR TENDENCIES IN KNOWLEDGE POLITICS}

I move now to a perspective which can incorporate - and make meaningful - a number of trends and analytical elements in knowledge production and knowledge governance, trends that are not necessarily compatible and which should be accounted for separately - but which together form the foundations for research policies:

- the importance of research for state formation;

- the growing significance of metagovernance;

- the alignment of research and economic growth (marketization); and

- the connection to societal processes (Box 1.2).

The first trend concerns the relationship between research and the formation and constitution of states. Research policy is, I argue, an integral aspect of state formation - that is, the process of forming and stabilizing a nation, its political institutions and its articulation with social and economic interests therein (Agar 2016). The field of state formation studies is vast and varied; suffice to say in this context that it has expanded from a focus on the interplay between social conflict and institution-building (Poulantzas 1978) to issues such as culture, language and identity in the stabilization of a state (Steinmetz 1999). State formation thus points to the complex ensemble of processes that together constitute a state, and the interplay between social, cultural, economic and political forms therein. 


\section{BOX 1.2 ANALYTICAL THEMES}

State formation: types of interaction between social, cultural, economic and cognitive (knowledge) forces in the constitution of the state. The aim is to outline the significance of science, technology and innovation to the current and future directions of the state.

Metagovernance: the composition of knowledge politics and the alignment between research policy and other relevant forms of state policy (such as foreign policy, defence, energy, higher education, the environment).

Connections to the mode of growth: how knowledge policies influence and are influenced by the dominant form of economic growth.

Connection to social forces and the citizenry: the social imageries and the forms of dialogue and deliberation with citizens of a state, including forms of advice, expertise and directionality.

Arguably, research and knowledge are important components of state formation, shown not only in the expansion of research policy as a field but also, and primarily, through its alignment with other aspects of state policy, with and for economic growth, and as part of the constitution of social relations and cultural identities in society. In analysing the dynamics of research policy, we should therefore be looking at how it resonates and intersects with the processes of state formation - especially in a historical period where the state is being challenged by the globalization of economic, social and political relations. Research governance serves as an instrument not only to stabilize national relations and national social and political directions, but also to enhance national visibility and position in global networks and relations.

The second trend - metagovernance ("the governance of governance") - concerns how the modern state is composed and how it aligns different tasks and missions in the governance and regulation of society. The state operates at the intersection of different forces - including geopolitical, functional and territorial - and is itself composed of different functions, organizations and missions (Jessop 2016). The contemporary state is, following Jessop, shaped by internationalization, destatization and denationalization. This means that the state is increasingly dependent on other forces and actors to realize its goals, and in the sense that national policies have to engage with processes and forces that reside outside the state's geographical demarcations.

A focus on metagovernance entails a how research policy aligns with other parts of the state - for instance policies for higher education, innovation or foreign relations - in addition to the connections to defence 
and energy as domains of national security and concern. While this would mean that the coherence of research as a specific policy field is undermined, if metagovernance (within and beyond national demarcations) is successfully institutionalized, it could also mean that its remit and influence are actually enhanced within a policy for knowledge - "knowledge policy", a term coined by Bell (1973) (see also Stehr 1994). Knowledge policy is a central concept to this study, as it expands the remit of research policy in the strict sense (funding and governance of public research) to show its interdependencies and systemic linkages, as well as highlighting the coordination problems that ensue when different policy domains are aligned. A focus on metagovernance thus also points to the complexities of state formation and the political dynamic that ensues when different societal areas and domains are orchestrated to interact.

The third trend is the marketization of knowledge and its importance for economic growth and competitiveness (Mirowski 2011; Slaughter and Leslie 1997). Knowledge has always been mobilized for political and economic purposes, but these purposes have now become explicitly articulated: research should reinforce and underpin the competitiveness of nations and other geographical entities, and firms and individuals within them; and policies should align the cognitive aspects of research with economic exchange. However, the forms of this alignment are not uncontroversial or given: they need to be configured and reconfigured, depending on policy traditions and trajectories, economic sectors, maturity of knowledge and technology, and the structure of markets - as well as the potentially conflicting interests between the public and private aspects of knowledge.

These relations have sometimes been framed in terms of systemic relations - "national systems of innovation" is the most common version (Nelson 1993). While such framings forcefully emphasize the many interdependencies of research and knowledge within (capitalist) economies, they tend to posit that such linkages are productive and useful, and that systems productively reforge relations between actors and organizations (cf. Caraça and Mendonça 2009). Stressing the ultimately (knowledge) political foundation of systemic interaction indicates that systemic relations need to be animated, and that they are always unstable and temporary.

The fourth analytical element is how scientific knowledge has emerged as an arena of deliberations and negotiations over power and influence in society (Jasanoff 1990). This would involve issues like reflexivity and how research contributes to the empowerment - and domination - of individuals and groups; but the most important aspect for our purposes is 
the relationship between research policy and social interests. This entails how knowledge is made legitimate (or not) in processes of negotiations and interaction with the citizenry and their various representatives, and how dominant discourses on the role of science in society are shaped and acted upon (Kuhlmann and Rip 2018). While this, potentially, would open up research to open-ended debates on the veracity of scientific claims and practices, making research governance a perpetually conflictual field with contradictory instruments and interests, it may also strengthen how knowledge is used as an instrument for social, economic and political purposes; hence the importance of such deliberations and engagements for the direction of knowledge policy.

\section{NARRATION AND INTERPRETATION: METHOD, MATERIAL AND APPROACH}

This book covers wide analytical (and geographical) areas, which has necessitated an eclectic empirical approach. I have relied heavily on secondary (and sometimes also tertiary) sources, but refinements of primary data form the bulk of my empirics. I have also, however (as far as possible), sampled official documents to illustrate policy standpoints and debates based on leads provided in other studies - documents that I have identified and procured myself or that were provided to me by interviewees. The use of secondary and tertiary sources is a recurrent and sometimes conflictual theme in the macrosociological literature: can sociologists really reconstruct past events and rely on historical methods to ascertain and "test" sociological assumptions on matters such as class, institutional set-ups or other social factors (cf. Mann 1994)? My response is, perhaps not unsurprisingly, affirmative: if we are to study the evolution of governance forms in many countries (and even in one country), we need to rely on data that has been collected and refined by others, perhaps also in forms that we cannot fully control or digest. Otherwise, comparative and historical studies would not be possible. Data elicited and refined by others should of course be treated with caution and, if at all possible, matched with similar studies, and the interpretative judgements and guiding devices made clear to the reader.

Documents only reveal parts and aspects of how policies are made and acted upon; not everything is documented, and not everything that is documented can be taken at face value. The analytical challenges of qualitative document analysis are numerous; this not only calls for rigour but also for maintaining the necessarily interpretative nature of analysing documents. The interplay between author and reader is critical, as are 
clearly and explicitly defined analytical choices, ways of organizing the material, and reasons for affording specific interpretations (Lincoln and Guba 1985; Manheim et al. 2010). Such rigour therefore depends on the capacity of the author to organize and structure the material in a narrative where chronological accounts are anchored in a contextual framework (White 1987).

To enable the formation of such a narrative, interviews may serve several purposes, including to contextualize data and to provide information and interpretations on specific events or processes. For all countries in this study, I conducted interviews and used them for two purposes: to introduce and discuss the policy systems as such; and to elicit information pertaining to specific policies or events. The interviews (45 in total) were mostly of an "elite" nature, with their own specific methodological concerns and challenges, such as access, engagement and feedback (Harvey 2011). The selection of elite interviewees was further complicated by the fact that they were spread over five continents, so in some cases I relied on the assistance of embassies or similar entry points for the provision of access. "Elite" in this context denotes government ministers and other senior officials, agency directors, policy advisors and holders of similar positions. To enable information access, I promised the interviewees anonymity and made notes, not tape recordings, of conversation-like interviews (cf. Aberbach and Rockman 2002). In some instances, this was critical due to prevailing political cultures of consensus where divergent and informal interpretations may be difficult to articulate, or when interviewees made interpretations of policy events that were not fully aligned with those of their patrons. In other cases, anonymity was not called upon but nevertheless seemed to ease conversations, allowing the interviewees to be frank and open about wideranging issues.

This book affords a narrative of the evolution of "knowledge politics", the goals it sets out to achieve and the contradictions it sets out to resolve, and how policies articulate with the social, economic and political contexts in the countries under study. Interviews, policy documents and secondary or tertiary sources constitute the empirical material. I have aimed not only to form a coherent narrative for each country on these bases, but also of the general evolution of nation-states as a response to the governance of knowledge over time - an "institutional narrative" (Hull 1975). It has been suggested that sociologists should adopt the practice of historians, namely to deploy narrative analysis as a means to anchor theoretical assumptions in rich historical material (Franzosi 1998). This aligns not only with the classic sociological studies of how science and technology reformed societies in the 19th century - 
with only blurred distinctions between the "factual" and the "literary" aimed at deciphering the interplay between purposeful action and structural factors (Lepenies 1988; Turner 1999) - but also with more recent studies of frictions in the constitution of society. Such frictions and rifts are difficult to pinpoint and elevate without an underlying narrative (Kumar 1978; Rodgers 2011; Bell 1976).

Texts and narratives have indeed been subject to rising interest among social scientists in the last decades as part of the "linguistic turn", with its emphasis on texts as socially constitutive properties (for a prominent example from the social studies of science, see Myers 1990). I do not consider the present study to be more than indirectly related to the linguistic turn; the interviews and texts are not seen as forces in and unto themselves, but rather as expressions of power, resource mobilization and the enactment of policy networks, with purposeful action as a foundation.

My intention with this narrative analysis, in the manner of historians, is to organize and structure historical material with an orientation towards revealing broad patterns (along the lines outlined above on state formation, metagovernance and socio-economic alignment), via a narrative account formed on the basis of a reconstruction of narratives in texts and interviews (Polletta et al. 2011). Hence, I will mix materials to outline these patterns and to highlight how history has evolved and how current policy choices and directions may be understood in relation to earlier events and periods. The chapters that follow will therefore be structured along the analytical themes outlined above (and summarized in Box 1.2), weaving together a relatively loose analytical framework with country narratives to enable historical accounts that make sense of the choices and directions of knowledge politics in vastly different countries, but also allowing for comparisons in time and space.

Following this brief theoretical and methodological exposé, the following chapters present an overview of how research policies have evolved in the ways outlined above in different countries and contexts. The chapters are based on national narratives of: how research policies have been aligned with nation-building and state formation; how research policies have been enmeshed in metagovernance; how they relate to economic growth; and how they articulate with societal processes - in all, how knowledge policies have been forged in their specific historical and geographical settings. 